\title{
High repetition rate multi-similariton laser
}

\author{
Auro M.Perego ${ }^{1, *}$, N.Tarasov ${ }^{1,2}$, Kestutis Staliunas ${ }^{3,4}$ and Sergei K.Turitsyn ${ }^{1,5}$ \\ 1. Aston Institute of Photonic Technologies, Aston University, Birmingham, B4 7ET, UK \\ 2. Institute of Computational Technologies, SB RAS, Novosibirsk 630090, Russia \\ 3. Departament de Física i Enginyeria Nuclear, Universitat Politècnica de Catalunya, Rambla Sant Nebridi 22, 08222 Terrassa, \\ Barcelona, Spain \\ 4. Institució Catalana de Recerca i Estudis Avançats, Pg. Lluis Companys 23, 08010, Barcelona, Spain \\ 5. $\quad$ Novosibirsk State University, Novosibirsk 630090, Russia \\ "peregoa@aston.ac.uk
}

We present the numerical demonstration of an harmonically mode-locked multi-similariton laser supporting a low jitter, stable train of self similar high repetition rate pulses exploiting, as mode-locking mechanism, the principle of dissipative Faraday instability (DFI) induced by zigzag modulation of spectral losses $[1,2]$. At variance with the theoretical and experimental studies on the DFI $[1,2]$, where the amplification was distributed along the fiber, we propose here a lumped amplification scheme suitable for a more flexible design of mode-locked lasers pumped by rare-earth gain medium (Erbium, Ytterbium). We have considered an unidirectional all normal dispersion ring resonator with two lumped amplifying sections separated by two passive nonlinear dispersive fibers. Just before each amplifying section is located a spectral filter. The two filters differ by having the transmittance profile respectively blue- and red-detuned relatively to the amplifiers central frequency. The detuned spectral filters provide the necessary periodic zigzag modulation of the spectral losses needed to trigger the DFI. The CW operation of the laser is unstable and the growth of spectral sidebands results in a temporal modulation of the field temporal profile leading to the formation of a pulse train with repetition rate corresponding to the instability frequency around $0.1 \mathrm{THz}$ and pulse duration of about 3 ps. Propagation in the fibers has been modeled using the generalized nonlinear Schrödinger equation and the lumped amplification by a saturable gain term with spectral bandwidth typical of rare-earth amplifiers.

(a)

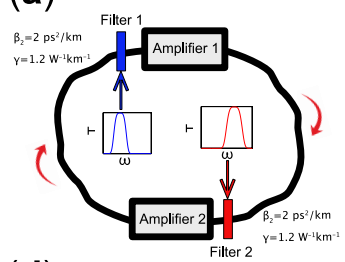

(d)

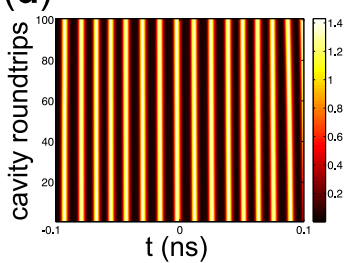

(b)

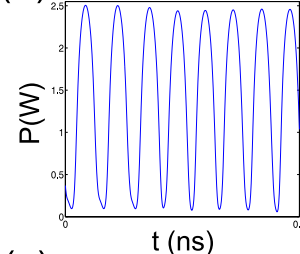

(e)

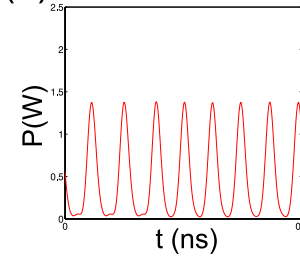

(c)

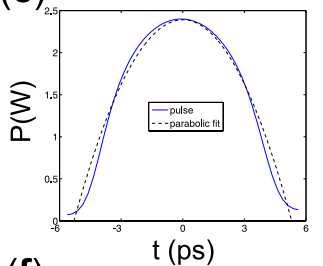

(f)

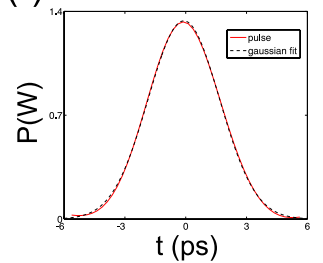

Fig. 1 The laser setup (a). Pulse train before filtering (b), and parabolic fit of the individual pulse (c). Pulse train after filtering: roundtrip evolution (d), intensity profile (e) and gaussian fit of the individual pulse (f).

The suggested scheme demonstrates clearly that lumped amplification can be used to achieve modelocking via DFI allowing for improvement in high power ultrafast laser design [3]. The features of the resonator can be easily modified resulting in a tunability of the repetition rate by 2 orders of magnitude flexibly depending on power, dispersion, nonlinearity and fiber length. At variance with regenerative similariton lasers [4], despite the resemblance of the setup, the pulses generated are not randomly distributed in time, but result in a regular low jitter train with obvious advantages in practical applications.

\section{References}

[1] A. M. Perego, N. Tarasov, D. V. Churkin, S. K. Turitsyn and K. Staliunas, "Pattern formation by dissipative parametric instability", PRL 116, 028701, (2016).

[2] N. Tarasov, A. M. Perego, D. V. Churkin, K. Staliunas and S. K. Turitsyn, "Mode-locking via dissipative Faraday instability", Nat. Commun. 7, 22441, (2016).

[3] A. Chong, L. G. Wright and F. W. Wise, "Ultrafast fiber lasers based on self-similar pulse evolution: a review of current progress", Rep. Prog. Phys. 78,113901 (2015).

[4] T. North and C. Brès, "Regenerative similariton laser" APL Photonics 1, 021302 (2016). 